\section{Hera: The Fundamental Strong Interaction Under the Microscope}

Robert Peschanski

Service de Physique Théorique, Saclay, Gif-sur-Yvette, France

The Hera accelerator at Desy, Germany's national particle physics laboratory at Hamburg, is the equivalent of a gigantic microscope that scrutinizes the $10^{-18} \mathrm{~m}$ substructure of the proton, ie quarks and gluons, the elementary quanta of the fundamental strong interaction. Experimental data on deep inelastic reactions and theoretical expectations of Quantum Chromodynamics, the modern quantum field theory of quarks and gluons are discussed. Recent findings and unexpected phenomena observed in the Hera detectors are also reported.

\section{A Gigantic Microscope}

Hera is a high-energy particle accelerator. Its unique feature is that it works as an asymmetric collider since both projectiles (leptons: electrons or positrons) and targets (protons) are accelerated in two beams, but to different energies: $27.6 \mathrm{GeV}$ for the leptons and $820 \mathrm{GeV}$ for the protons. By comparison LEP at CERN in Geneva uses two symmetric lepton beams of $45 \mathrm{GeV}$ and (now) more than $80 \mathrm{GeV}$ each, and the Tevatron at Fermilab in the US works symmetrically with protons against antiprotons of $900 \mathrm{GeV}$ energy each. The lepton and the proton beam at Hera are designed to collide in two intersection zones. At these points, two big detectors, called $\mathrm{H}_{1}$ and ZEUS, analyse the information coming from the collision and detect the particles produced.

Why does Hera act as a gigantic electronic (or positronic) microscope deep inside the proton? The answer lies in the quantum properties of the photon which is the messenger of the electromagnetic interactions between the lepton and the proton. When an electron approaches the proton its trajectory is deflected and it emits a photon which is absorbed by the proton (fig 1 ). However, this photon is virtual, which means that it is located only

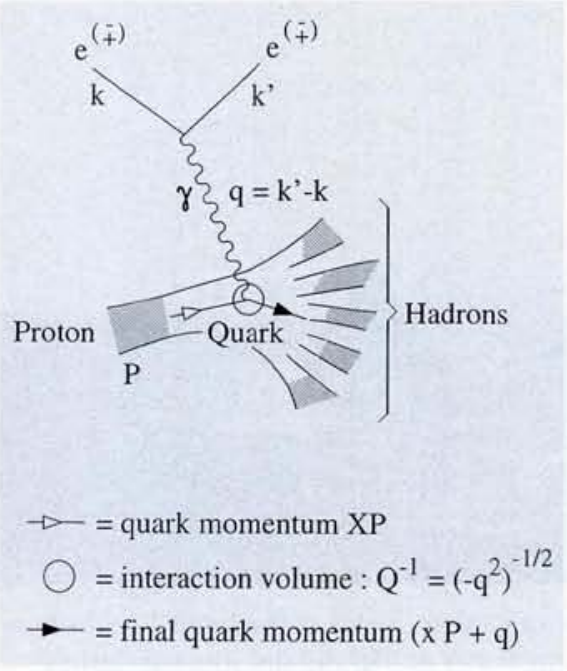

Fig 1 Deep-inelastic e-proton reaction The electron or positron $\mathrm{e}^{( \pm)}$interacts with the proton by emitting a virtual photon $\gamma$ with momentum $q$. The quark with momentum fraction $x$ of the proton momentum $p$ absorbs the photon in a point-like interaction. The interaction "volume" of size $1 / Q$ is represented by a small circle, in which the quark moves almost freely

in a small space-time area inside the interaction region and does not materialize as a real photon. This quantum mechanical process, which associates interactions with virtual particle exchange, is the basis of the exploratory potential of an accelerator like Hera. Indeed, if the energy conveyed by the virtual photon from the electron to the proton is large enough, the interaction area can reach a size as small as $10^{-18} \mathrm{~m}$ at Hera, $i e$ a thousandth of a fermi, one fermi being the typical proton size. Previous leptonproton experiments reached a tenth of a fermi and yet were powerful enough to reveal the quark and gluon substructure of the proton. The interesting thing about Hera is that for the first time it opens a resolution window of two more orders of magnitude inside the proton. (The improved resolution recently led to a spectacular new development: more events than expected from the standard model have been found at the smallest attainable scale, see EN 282 March/April 1997.)

Let us elaborate somewhat on the extraordinary features of Hera as a microscope and consider what is usually called deep inelastic scattering ( $f$ ig 1 ). In such a process, the lepton transfers momentum $q$ to the proton. This photon acts as a probe inside the proton with a wavelength of the order

$$
\left|q^{2}\right|^{-1 / 2}=1 / Q
$$

In fact, the value of $Q$, ie the resolution of the microscope, crucially depends on the amount of energy which can be released in the collision, and thus on the kinematic features of the lepton deflection, namely the scattering angle and the energyretained by the outgoing lepton in the laboratory frame.

Now the key feature of the game: let us assume that the high energy proton is made of point-like particles, the partons, freely moving inside the proton. Among them there are electrically-charged ones, the well-known quarks and anti-quarks, which are able to interact with the photon probe. Thus the photon hits a quark carrying a fraction $x$ of the total momentum $(\mathrm{Q} / \mathrm{M})^{2} \cong 10^{5}$. The point-like quark (or anti-quark) shows the same behaviour as the lepton, namely it is deflected but not 'destroyed' by the photon, as is the case for a compound particle. Indeed, once the quark is expelled by the interaction, the proton does explode into numerous particles for most of the time. One then writes an equation, following the momentum conservation law

$$
(x p)^{2}=(x p+q)^{2} \Rightarrow x=\frac{-(q)^{2}}{2 p q}
$$

reflecting the identity of the quark masssquared before $(x p)^{2}$ and after $(x p+q)^{2}$ the interaction with the photon ( $\left(f_{i} 1\right)$. The final result is crucial since it relates the (microscopic) momentum of the struck quark $x$ to the (macroscopic) kinematics of the reaction which can be driven by the experimentalists. Indeed, one may rewrite the right-hand side of formula 1 as

$$
x=\frac{Q^{2}}{W^{2}-M^{2}-Q^{2}}
$$

where $Q \equiv\left(-q^{2}\right)^{1 / 2}$ is the inverse wavelength of the photon, $W^{2} \equiv(p+q)^{2}$ is the total centre-of-mass energy of the photon- 
proton system and $M \sim 1 \mathrm{GeV}$ is the proton mass. The 'magic' of formula 1 is that by exploring the kinematics of the reaction one is able to investigate the proton microscopic content in a large region of $x$, ie of the momentum spectrum of its inner fundamental constituents.

In fact the $(x, Q)$ variables, or more precisely the $\left(\ln 1 / x ; \ln \left(Q^{2} / M^{2}\right)\right)$ plane, defines for us the natural field of investigations of the structure of the proton. Due to the large energies of both the proton and the lepton beams, this domain can reach values of the order

$$
x \cong 10^{-5} ; \text { and }\left(\frac{Q}{M}\right)^{2} \cong 10^{5}
$$

Thus, varying both the size-resolution $1 / \mathrm{Q}$ of the probe and the region $x$ in the energy spectrum of the constituents, the scientist has at his or her disposal a magnificent microscope for looking inside the proton. So, what did we expect to see and what has appeared in the first three years of operation at Hera?

\section{The Fundamental Strong Interaction}

Long before Hera, the study of leptonhadron or nucleus interactions at small distances ( $Q$ large) has been a basic tool for the investigation of the structure of hadrons and strong interactions. The first elementary constituents of hadronic matter to be experimentally discovered, the quarks, were revealed by deep-inelastic reactions observed at SLAC. They showed the existence of nearly point-like particles inside the proton. Intriguingly enough, these constituents behave like free particles at short distances inside the hadron, while they are so tightly bound at larger distances that they never show up in detectors. This apparent paradox corresponds to a theoretical property of the fundamental strong interaction, the asymptotic freedom. In practice, the experimental determination of the density of constituents in the proton is extracted from the number of events registered in the detector as a function of small intervals of $x$ and $Q^{2}$. This allows the so-called structure function $F_{2}\left(x, Q^{2}\right)$ of the proton to be measured, which, in turn, is related to the density spectrum of quarks (and antiquarks) with fraction $x$ of the proton momentum, observed in a space-time interval $1 / Q$. More information can be obtained from an analysis of the hadronic final states, or from the analysis of the contributions coming from the polarization states of the virtual photon.
Let us now introduce the predictions of the theory of strong interactions for the structure function in the range covered by Hera.

In the quantum field theory of quarks and gluons called Quantum Chromodynamics (QCD), the elementary quantum fields associated with the quarks and gluons carry a new quantum number, colour. Indeed, besides mass and spin each quark or gluon is labelled by a colour index corresponding to an internal symmetry group. This symmetry is hidden, ie every measurable quantity must be colour-blind (or more precisely, correspond to a singlet of the colour group). This group $S U_{c}(3)$, has many far reaching implications. For instance, quarks may appear only in bound $q q$ or $q q q$ states which correspond to mesons or baryons respectively. Also, being a symmetry group in each point in space and time it implies that the interaction between quarks is conveyed by a set of 8 well-defined quantum states, the gluons. This is the principle of the so-called gauge field theories, which are the unique powerful theoretical tool for describing fundamental interactions among particles, including strong, electromagnetic and weak interactions, in a framework now called the 'standard model'. However, a noticeable peculiarity distinguishes QCD from the other gauge theories of the standard model, namely the property of asymptotic freedom, as mentioned before.

Contrary to photons, gluons can interact with themselves in consequence of the colour group structure. Indeed, via quantum fluctuation effects the gluon selfcoupling introduces a dependence of the interaction strength with the resolution scale $Q^{2}$. Thus the coupling strength depends on the resolution power of the probe. At short distances, the interaction becomes weak, quarks and gluons reach the asymptotic freedom regime where they become independent free particles. In contrast, at long distances, in particular at macroscopic observational distances, the interactions become stronger and stronger, leading to the non-observability of isolated quarks and gluons. This is called the confinement mechanism. In practice, QCD predicts a well-defined pattern of evolution of the density of quarks (and gluons) when one modifies the interaction size, which means (in more mathematical terms) QCD predicts evolution equations for the structure function $F_{2}\left(x Q^{2}\right)$ and $F_{G}\left(x, Q^{2}\right)$ for the gluons as a function of $Q^{2}$. This represents the basic prediction of the quantum field theory of quarks and gluons in the deep-inelastic domain.

In fact, the evolution equations predict that more and more gluons and 99 pairs are emitted, when $Q^{2}$ increases or $x$ decreases, at a rate determined by the fundamental constants of QCD. However, the validity of the QCD evolution equations in the Hera range was far from certain. Due to the very small value of quark

momentum ratio $x$ attainable at Hera, the exact theoretical 'apparatus' for deriving the evolution equations is not yet known. Intuitively speaking, the partons (quarks and gluons) are expected to proliferate so much that asymptotic freedom can be violated by a high density of particles. This is called the saturation phenomenon, where the density of produced quanta limits their mean free path. Many physicists were expecting to meet this saturation effect in the Hera experiments. It was really a challenge for Hera to see whether these predictions were realised or whether a different mechanism was taking place. Now comes the experimental verdict.

\section{The Verdict}

With the experimental results obtained from the 1993 and 1994 detection runs, knowledge of the quark and gluon structure functions has taken a considerable step forward. Deep aspects of the dynamics of strong interactions have been revealed, which have been discussed in numerous theoretical papers since then. In fig 2 , one may see data corresponding to a large coverage of the $\left(x, Q^{2}\right)$ domain for the proton structure function $F_{2}$. Data from both $\mathrm{H}_{1}$ and ZEUS experiments are plotted as a function of $x$ for a large range of $Q^{2}$. As a first qualitative statement, it is clear that the structure function, and thus the density of quarks and antiquarks in the proton, increases quickly when one reaches very small fractions $x$ of the proton momentum. The proliferation phenomenon, as predicted by the QCD evolution equations, is at work in the Hera range. The saturation limit does not seem to be reached, or is not required by the data (yet it cannot be excluded). A second feature of this proliferation mechanism is that it becomes stronger when $Q^{2}$ is larger. This second feature is also well-supported by the expectations from the QCD evolution equations, since in the small $x$ regime it extends the so-called scaling violations, corresponding to the deviations from free partons due to quark-gluon interactions already studied at lower energy. This phenomenon is related to the fact that increasing the resolution power of 


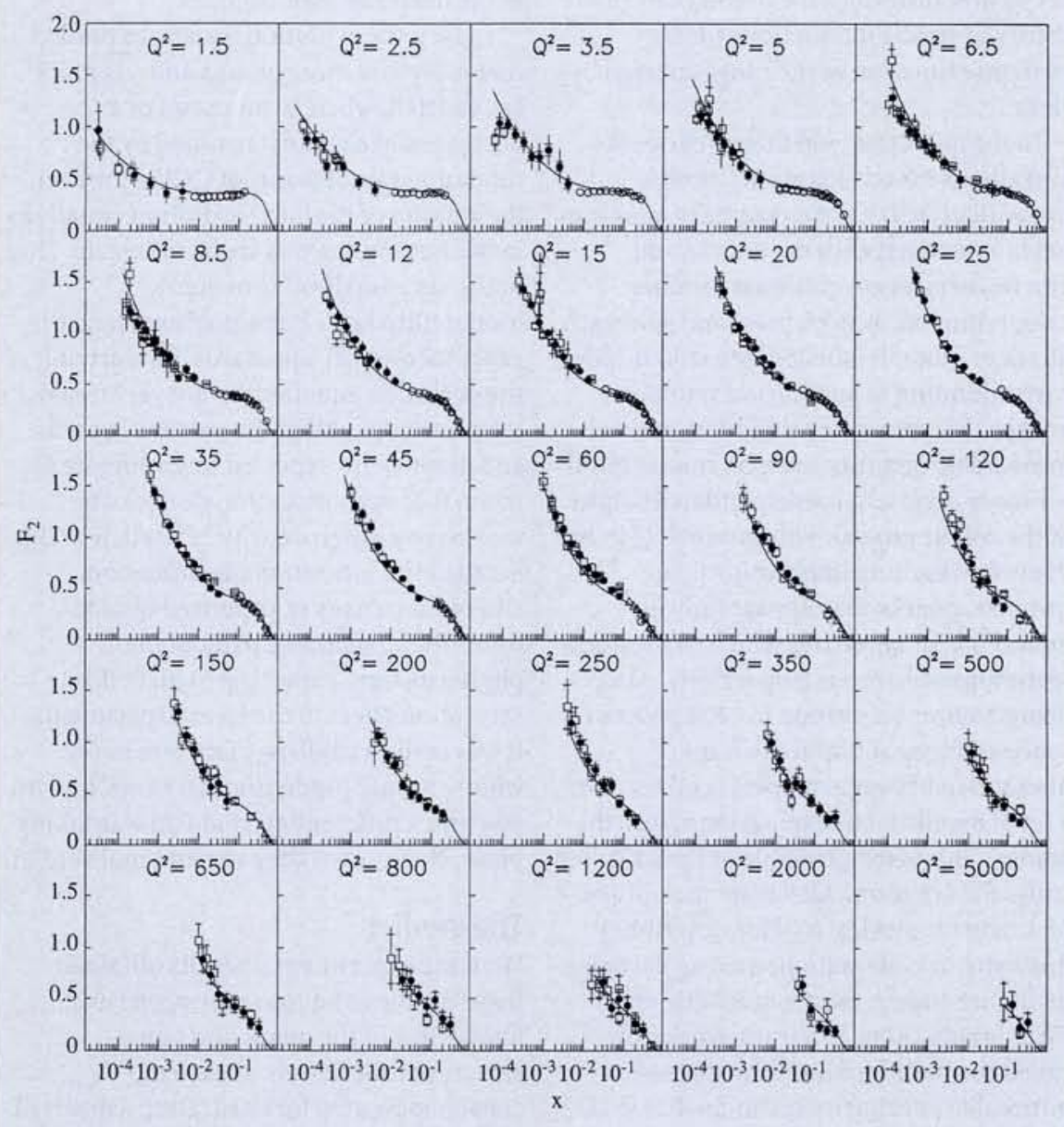

Fig 2 Experimental results for the quark-singlet structure function The 1994 published data from both $\mathrm{H} 1$ and ZEUS collaborations $[1,2]$ are reported as a function of $x$ for different values of $Q^{2}$. The continuous line represents the theoretical fit based on the Gluck-Reya-Vogt model [3] using the QCD evolution equations

the probe by increasing $Q^{2}$ allows one to separate the struck quark from the surrounding gluon 'cloud' due to quantum field fluctuations. It is thus clear that the results from Hera are telling us something about the deep mechanisms at work in the fundamental strong interaction. The experimental tests are still going on. In particular some data at small values of $Q^{2}$ down to $0.16 \mathrm{GeV}^{2}$ are being analysed, with the interesting issue of examining the transition between deep-inelastic and reactions initiated by quasi-real photons (Q 0).

The theoretical studies using the $Q C D$ evolution equations are too numerous to be discussed in this general article. In order to give a very brief survey on the theoretical descriptions, we show in fig 2 (continuous line) the interesting description in terms of a model, Gluck-Reya-Vogt (GRV). In fact, the assumption of the GRV model is that the $\mathrm{QCD}$ evolution equations are valid in a very large domain, much bigger than the one initially expected for the structure functions. The GRV model has anticipated the observed rise in the quark densities. However, discussions are still going on and many points of view are present among theoreticians concerning the $\mathrm{QCD}$ treatment of the structure functions. We will in particular see that there is not only one but two different classes of evolution equations.

If one knows about quarks and antiquarks in the proton, what can be said about gluons? In a direct way it is difficult, since the gluons carry no electric charge and thus cannot be probed by the virtual photon. However, there are a few indirect ways to detect them and examine their density distribution in more or less the same kinematic range as for the quarks. Gluons can be responsible for jets observable in the structure of the final states after a deep-inelastic scattering event. In a more indirect, but more precise way, one uses the connection between the $Q^{2}$ evolution of the quark structure function and the gluon distribution to obtain an estimate of the density profile of the latter based on the fit to $F_{2}$. In $f_{i g} 3$, we show the result obtained by a fit using data from $\mathrm{H}_{1}$ and the fixed-target experiment NMC. The price paid for this indirect determination is some uncertainty represented by a shaded area in fig 3 . In fact, these results confirm the proliferation mechanism, valid for gluons as well as for quarks. At fixed $Q^{2}$ the gluon density increases with decreasing momentum fraction $x$. As a function of $Q^{2}$ the proliferation increases, revealing large gluon scaling violations when $Q^{2}$ increases.

It is quite intriguing that at least two different classes of $Q C D$ evolution equations exist, being candidates to explain the observed features in the domain of small $x \leq 10^{-2}$. For instance, the grey area shown in fig 3 was obtained by using the basic Dokshitzer-Gribov-Lipatov-AltarelliParisi equations (DGLAP) which are based on the $Q C D$ predictions for the $Q^{2}$ evolution. A very different approach, due to Balitsky- Fadin- Kuraev- Lipatov (BFKL), is based on the known $Q C D$ properties of the $x$-evolution. In fig 3 , the BFKL type of prediction is depicted by a continuous curve fitting the data well in the region of $x$ $\leq 10^{-2}$. There is thus no easy way at the moment to determine which of the evolution equations is more adequate. One challenge for future experimental investigations is to look for the favoured equation, helping the theoreticians to understand the fundamental features which predominate among the different treatments of $\mathrm{QCD}$. A hope for the future measurement of $R \equiv F_{L} / F_{T}$, the longitudinal to transverse polarization ratio of the virtual photon. (Note that a real photon has only transverse polarizations). Indeed, contrary to the predictions for the gluon structure function, a sizeable difference for $R$ could appear between the two competing $\mathrm{QCD}$ evolution equations. Another active issue is the study of the associated final hadronic states.

On theoretical grounds, the ultimate hope is that deep-inelastic scattering experiments at Hera will help us better understand QCD as an established theory of the fundamental strong interactions. Here are two questions which are now being discussed among the specialists:

a) If parametrizations from the weakcoupling regime (like GRV or similar rival $Q C D$ analyses) are able to describe well the data in a large range of $\mathrm{Q}^{2}$ and $1 / \mathrm{x}$, how will the strong-coupling regime of $Q C D$ manifest itself? 

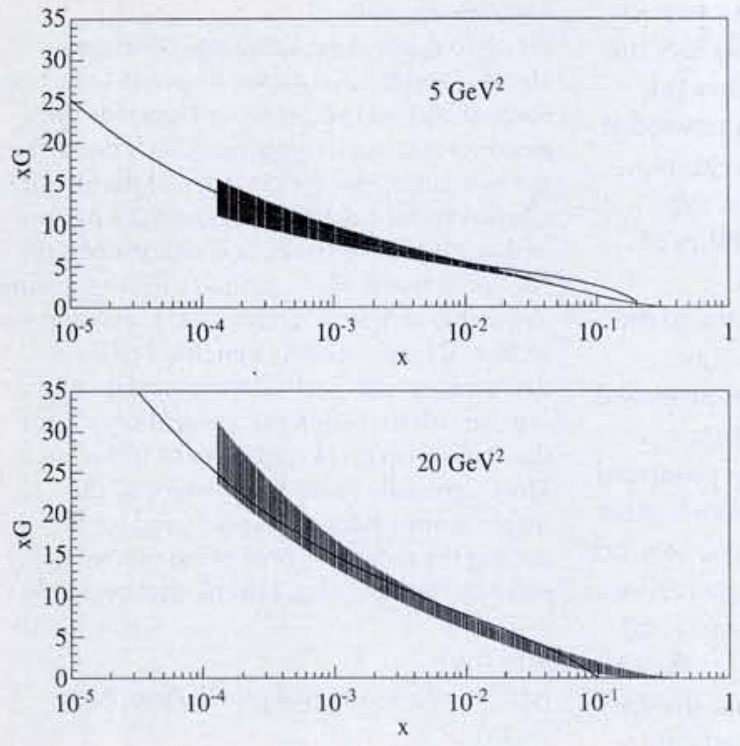

Fig $3 \mathrm{H} 1$ evaluation of the gluon structure function The shaded area represents the estimate of the gluon structure function $F_{G}$ for $Q^{2}=5$ and $20 \mathrm{GeV}^{2}$. It is obtained from $\mathrm{H} 1 \mathrm{fit}$ of $\mathrm{F}_{2}$ and the use of the $Q^{2}$ evolutions equations (in the second-order approximation of the perturbative expansion). The continuous line represents the predictions at $x<10^{-2}$ for the $x$ evolution equations for the same structure function
New preliminary data on the structure function at low $Q^{2}$ indicate a transition between the two regimes, but it is difficult to find a satisfactory theoretical description. For instance GRV-like parametrizations suddenly fail when $Q^{2}$ decreases below a certain limit. On the contrary, parametrizations based on ordinary 'soft' hadron-hadron reactions are unable to describe the structure functions beyond this limit.

\section{b) If two and not just one unique QCD} evolution equation can describe the pattern of data, what does it imply for their respective validity range?

Indeed, these evolution equations are based on different resummation schemes of the perturbative expansion of the theory at weak coupling. As such, the theory is not used in a complete way which should include both weak and strong coupling. One way out would be to disprove one or other of the approaches.

However, their theoretical foundation and phenomenological success do not make the choice easy. Another way out is to look for unification of the DGLAP and BFKL evolution equations, valid in a large 2-dimensional $\left(Q^{2}, 1 / x\right)$ region. However, the equations are based on quite different properties of the perturbative regime of QCD. Some progress is expected from the calculations at next-to-leading order of the BFKL equation and from a unified description of the multi-gluon inelastic processes which build the structure functions.

On phenomenological grounds there is a need to perform precise predictions on the hadronic final states which are created in a deep-inelastic reaction. One expects significant differences between the final states contributing to DGLAP or BFKL evolutions. However, the discussion is obscured by the difficulty of relating gluonic (or quark) final states to the detected hadronic states.

More fundamentally, the problem involves the mysterious confinement phenomenon, ie the non-existence of quarks and gluons as free states at large distances. Indeed, a very old theoretical result, the so-called 'Froissart Bound', proves that cross-sections at very high energy (and by extension the structure functions at very low $x$ ) should not grow faster than logarithmically, contradicting the predictions of the evolution equations
- both of them. This fundamental result is based on the conservation of probabilities (called the 'unitarity' property) and the existence of a particle with a minimum mass, the pion (related to a mathematical 'analyticity' property). This pion mass can be regarded as a consequence of the confinement mechanism, as was shown by numerical simulations of the QCD interaction at strong coupling on a lattice representing the discretized 4-dimensional space-time. How 'unitarity' and 'analyticity' can be recovered in a theoretical scheme starting from the fundamental equations of QCD, is a real challenge for basic theoretical research in the future.

\section{A Surprise: 'Hard Diffraction'}

As a last topic of this brief review article let us introduce the puzzling problem of hard diffractive events. It came as a surprise that a sizeable fraction of deep inelastic events (of the order of $1 / 10$ ) has a quite unexpected feature: hard collisions are observed while no particle is registered in the forward detectors, ie in the line-offlight of the incident energetic proton beam. You may see a beautiful example of such an event in fig 4 registered by the ZEUS experiment. More precisely, it is now usual to speak of 'rapidity gap' events, which means that no particle is detected in a given angular aperture around the forward (proton) beam direction. The only possible experimental interpretation of diffractive events is that the proton remnants (or the recoil proton itself) have evaded detection in the beam pipe. Some recent experimental measurements nearer

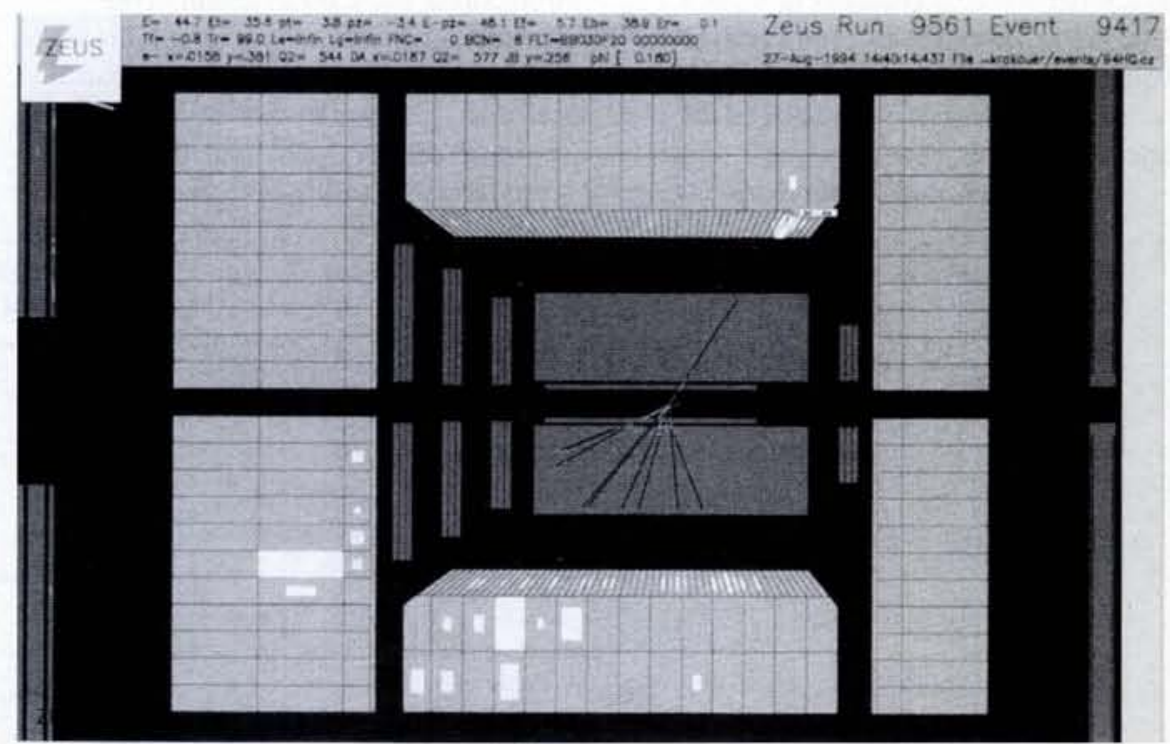

Fig 4 'Hard diffractive' event in the ZEUS detector 'Rapidity-gap' event inside the ZEUS detector. The forward region does not show any activity. The high $Q^{2}$ deflected electron $\left(Q^{2} \sim 575 \mathrm{GeV}^{2}\right)$ is on the upper right 
the forward direction have confirmed this statement by direct detection of the recoiled system. The existence of such events and their large contribution came as a big surprise since the hard collision corresponding to a deep-inelastic reaction (with a sizeable amount of energy released in the target) would naively imply that the final state should be a large number of hadrons scattered in a large phase-space region without an empty window in the forward direction. Nature is different. Why?

Among the very few theoretical ideas available, the proposal of a quark and gluon substructure of the Pomeron appears to be very promising. The Pomeron is the mysterious 'messenger' of elastic and diffractive interactions at high energies. Indeed, it has been recognized that a proton at high energy may interact quasielastically or diffractively, ie remaining as a proton state, resonances or low-mass systems, following the initial line-of-flight after the interaction. These processes are commonly called 'soft' by contrast with the deep-inelastic reactions called 'hard'. The soft interactions at high energy are thus attributed to the exchange of a composite object, the Pomeron - after the late Soviet physicist Pomeranchuk.

Let us assume that the Pomeron behaves as an ordinary hadron with a given substructure made of quarks and gluons. One is led to the picture depicted in fig 5 , where the Pomeron is probed by the photon in much the same way as the proton is probed in ordinary collisions (compare fig 5 with fig 1). This is the theoretical scheme proposed for the so-called 'hard diffraction' - 'hard' because it is initiated by a short-distance photon probe, and 'diffractive' because this probe acts on the Pomeron instead of the proton which remains itself weakly perturbed by the interaction (see fig 5).

However, this explanation relies on the

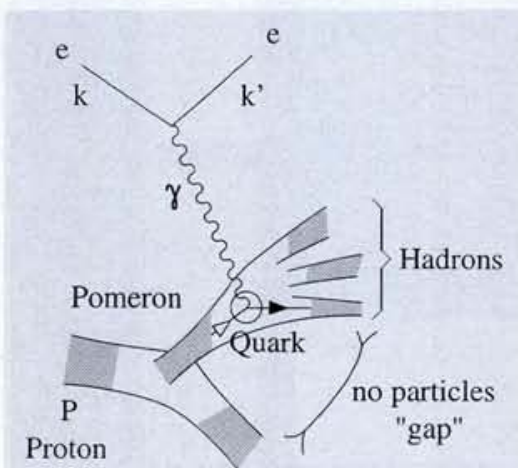

Proton

(recoil) assumption that the Pomeron is a particle like others. QCD, in its present state of the art, cannot easily prove or disprove the assumption. It would require a knowledge of the theory in the long-range regime, where asymptotic freedom and weak coupling are lost. Our present ability of doing calculations based on the fundamental theory is very limited in the long-range regime. Moreover a very different interpretation has been proposed which relies on the colour quantum number content of the final state produced in deep-inelastic scattering. Following this interpretation, there should not be so much difference between ordinary deep-inelastic scattering (without diffraction, fig 1 ) and 'hard diffractive' scattering (fig 5 ). The idea is to consider separately the short-distance interaction, which is supposed to be the same in both cases, and a long-distance rearrangement of the colour quantum number of the final state of hadrons following the line-of-flight of the initial proton. The argument is simple (while its concrete realization might be complicated). When the final state is coloured (ie nonsinglet of the colour group) the strong attraction of colourful states makes the proton 'explode' into many particles with no rapidity gap. On the contrary, if the outgoing system is colour-blind (coloursinglet), no interactions are felt by the system which remains in a low-excited state. For the present, neither interpretation has been favoured by the experimental results, nor the other models on the market. 'Hard diffraction' still deserves a convincing explanation. Thus we are exploring the heart of strong interactions, since the problem of the Pomeron has remained a true enigma for more than 40 years now. There is great hope that the direct investigations of the Hera-microscope will help to make substantial progress in that direction.

\section{Acknowledgments}

I wish to thank Andrzej Bialas (Krakow), Henri Navelet, Christophe Royon (Saclay) and Samuel Wallon (Saclay, then Desy) for the good research collaboration which nourished the content of this article. Special thanks go to $\mathrm{Ch}$. Royon for helping me to find the figures and numerous remarks and suggestions on the text. I thank M.-C. Lemaire for suggesting to me this article, A. Dauba and J. Beucher for technical help. I greatly benefited from discussions with Joel Feltesse (Saclay) and a mutual collaboration for a recent review for the staff of DAPNIA department in Saclay. More generally I very much enjoyed the whole atmosphere of research and work among the community of physicists interested in Hera physics, I thank them warmly.

\section{References}

[1] H1 Coll., S. Aid et al., Nucl. Phys. B470 (1996) 3.

[2] ZEUS coll., M.Derrick et al., Zeit. für Phys. C72 (1996) 399.

[3] M.Glück, E. Reya, A. Vogt, Zeit. für Phys. C67 (1995) 433.

[4] H. Navelet, R. Peschanski, Ch. Royon, S. Wallon, Phys. Lett. B385 (1996) 357.

\section{Bibliography}

A recent discussion and general review open to the future can be found in: Future Physics at Hera, Proceedings of the Workshop 1995/96 (G. Ingelman, A. De Roeck, R. Klanner Eds., www.desy.de/heraws96)

On the theoretical side, I recommend for the aficionados: Basics of Perturbative $Q C D$, by Yu.L. Dokshitzer, V.A. Khoze, A.H. Mueller and S.I. Troyan, Editions Frontières, (J. Tran thanh Van, Ed., 1991); Perturbative Quantum Chromodynamics, Advanced Series on Directions in High Energy Physics-Vol 5, Editor: A.H. Mueller (World Scientific Eds., 1989).

Other programmes of interest are actively under investigation at Hera, for instance charged current interactions, quasi-real photoproduction reactions, production of vector mesons and heavy quarks and searches for exotic particles. Soon the physics of $B$-mesons and their interaction with nuclei (using atomic jets) will be intensively studied at the accelerator.
Fig 5 Deep-inelasticePomeron reaction The electron or positron $\mathrm{e}^{ \pm}$ interacts by emitting a virtual photon $\gamma$ (with momentum q) while the proton is diffractively scattered, without being excited, and emits the would-be Pomeron. The quark assumed to be present in the Pomeron absorbs the photon in a point-like interaction. The 'hard' interaction volume of size $1 / Q$ is represented by a small circle

\section{STAFF SCIENTIST ANNOUNCEMENT ELETTRA (SINCROTRONE TRIESTE)}

The Sincrotrone Trieste, builder and operator of the ultrabright storage ring Elettra is seeking one or two outstanding candidates for its Experimental Division. We are looking for exceptional individuals. qualified and capable to develop indipendent programs for innovative exploitation of the unique performances of Elettra.

Suitable candidates should send a curriculum with a list of publications, a short (one and a half page) statement on their plans to exploit the brightness and coherence of Elettra and the names of three references (including address, telephone, telefax and e-mail) to:

Prof. Giorgio Margaritondo

Director Experimental Divisio

Sincrotrone Trieste

S. S. $14 \mathrm{Km} 163.5$ in Area Science Park

34012 Basovizza - Trieste

Italy

We emphasize that the candidates must provide evidence not only of the required technical background, but also of indipendence and creativity. Their field of research can belong to anyone of the areas of applications of Elettra, for example microscopy, holography, spectromicroscopy, cristallography, highresolution or time-resolved spectroscopy and circular polarization techniques. Knowledge of the Italian language is not required. We particularly welcome applications from outstanding women scientists. language is not required. We particularly welcome applications from outstanding women scientists.
Information about Elettra can be found at www.elettra.trieste.it.

The deadline for receiving the applications is 31 October 1997. 